\title{
BMJ Open Possible relationship between general and pregnancy-related anxiety during the first half of pregnancy and the birth process: a prospective cohort study
}

\author{
Johanna Maria Koelewijn, ${ }^{1,2}$ Anne Marie Sluijs, ${ }^{3}$ Tanja G M Vrijkotte ${ }^{4}$
}

To cite: Koelewijn JM, Sluijs AM, Vrijkotte TGM. Possible relationship between general and pregnancyrelated anxiety during the first half of pregnancy and the birth process: a prospective cohort study. BMJ Open 2017;7:e013413. doi:10.1136/ bmjopen-2016-013413

- Prepublication history and additional material are available. To view these files please visit the journal online (http://dx.doi. org/10.1136/10.1136/bmjopen2016-013413).

Received 12 July 2016 Revised 9 January 2017 Accepted 20 February 2017

\section{CrossMark}

${ }^{1}$ Sanquin Research and Landsteiner Laboratory, Department of Experimental Immunohematology, University of Amsterdam, Amsterdam, The Netherlands

${ }^{2}$ Department of Obstetrics and Gynaecology, Academic Medical Center, University of Amsterdam, Amsterdam, The Netherlands

${ }^{3}$ Department of Obstetrics and Gynecology, Leiden University Medical Center, University of Leiden, Leiden, The Netherlands ${ }^{4}$ Department of Public Health, Academic Medical Center, University of Amsterdam, Amsterdam, The Netherlands

Correspondence to Dr Tanja GM Vrijkotte; T.Vrijkotte@amc.uva.nl

\section{ABSTRACT}

Objectives The rate of interventions during childbirth has increased dramatically during the last decades. Maternal anxiety might play a role in the progress of the labour process and interventions during labour. This study aimed to identify associations between anxiety in the first half of pregnancy and the birth process, including any interventions required during labour. In addition, differences in the associations by parity and ethnicity were explored.

Design Prospective cohort study.

Setting Primary care midwifery practices and secondary/tertiary care obstetric practices in Amsterdam, participating in the multiethnic ABCD (Amsterdam Born Children and their Development) study (participation rate 96\%; response 8266/12373 (67\%)).

Participants Included were women with singletons, alive at labour start, with a gestational age $\geq 24$ weeks $(\mathrm{n}=6443)$.

Independent variable General anxiety (State-Trait Anxiety Inventory state) and pregnancy-related anxiety (Pregnancy-Related Anxieties Questionnaire (PRAQ)) were self-reported in the first half of pregnancy.

Outcomes Associations between both forms of anxiety and several indicators of the birth process were analysed. Subgroup analyses were performed for parity and ethnicity. Results The prevalence of high general anxiety (State-Trait Anxiety Inventory score $\geq 43$ ) and pregnancy-related anxiety (PRAQ score $\geq P 90$ ) were $30.9 \%$ and $11.0 \%$, respectively. After adjustment, in nulliparae, both general anxiety and pregnancy-related anxiety were associated with pain relief and/or sedation (OR for general anxiety $1.23 ; 95 \% \mathrm{Cl}$ 1.02 to $1.48 ; 0 \mathrm{R}$ for pregnancy-related anxiety 1.45 ; $95 \% \mathrm{Cl} 1.14$ to 1.85$)$. In multiparae, general anxiety was associated with induction of labour $(\mathrm{OR} 1.53 ; 95 \% \mathrm{Cl} 1.16$ to 2.03) and pregnancy-related anxiety was associated with primary caesarean section (OR 1.66; 95\% Cl 1.02 to 2.70). Associations were largely similar for all ethnicities.

Conclusions High levels of general and pregnancyrelated anxiety in early pregnancy contribute modestly to more interventions during the birth process with similar associations between ethnic groups, but with some differences between nulliparae and multiparae.

\section{INTRODUCTION}

The rate of interventions during childbirth has increased dramatically in recent
Strengths and limitations of this study

- We performed a prospective study in a large multiethnic cohort.

- We used validated questionnaires to assess both forms of anxiety.

- Anxiety was measured in the first half of pregnancy.

- The subscale for Fear of Childbirth (FOC) within the Pregnancy-Related Anxiety Inventory was not suitable to measure FOC in multiparous women. Therefore, our focus was on the total score on pregnancy-related anxiety and not on the subscales.

- Our data are collected in 2003 and 2004. Since 2004 the intervention rate has increased, but there is no reason to assume that the association between anxiety and (interventions in) the birth process has changed.

decades. For example, in the Netherlands, the caesarean section rate rose from $8.1 \%$ to $13.6 \%$ in 1993 to 2002 , and $16.7 \%$ in $2010 .^{12}$ The rate of labour induction increased from $15 \%$ to $21 \%$ during 2008-2013. ${ }^{3}$ Although the absolute incidence of caesarean section is higher in other western countries compared with the Netherlands, a similar rise in caesarean sections occurred in other western countries. $^{4-7}$

The progression of the birth process and concomitant interventions are associated with maternal characteristics such as age, parity, body mass index (BMI), ethnicity, illness and infant birth weight, as well as with organisational factors such as existing guidelines, the availability of 24 hours pain relief, the profession of the obstetric care provider (midwife vs physician) and the level of care (primary/secondary). ${ }^{8-12}$ Moreover, maternal anxiety might play a role in the birth process. Although one review found no overall association between anxiety and obstetric complications, specific types of anxiety (such as fear of childbirth) may be 
associated with specific complications and interventions, such as prolonged labour and caesarean section. ${ }^{13}$

Several studies have shown a relationship between fear of childbirth and duration of labour, ${ }^{14-16}$ epidural analgesia $^{17}$ and elective ${ }^{18-22}$ and emergency caesarean. ${ }^{1523}$

On the other hand, other studies reported no such relationships. ${ }^{24-26}$ One explanation for these inconsistencies could be differences in cultural, social and organisational characteristics between countries. These factors can mediate or exacerbate the effect of anxiety on the birth process and on concomitant interventions. For example, some ethnic groups are more susceptible to stress-induced neuroendocrine and inflammatory pathways, which could lead to adverse perinatal outcomes. ${ }^{27-30}$ Also, cultural and social differences between ethnic groups (eg, language barriers, unfamiliarity with the obstetric care organisation) may explain differences between ethnic groups regarding the influence of anxiety on the birth process. ${ }^{31-34}$ In addition, it is unknown whether the association between anxiety and the birth process differs between nulliparous and multiparous women as well as between women giving birth in primary and secondary care. To our knowledge, no study has investigated the level of care and ethnicity in the association between general and pregnancy-related anxiety, and the progression of birth.

Anxiety in pregnancy is associated with shorter gestational age and has negative implications for fetal neurodevelopment and child outcomes. ${ }^{35}$ Women with high fear of childbirth in pregnancy are at risk for psychiatric problems postpartum, for example, postpartum depression and even post-traumatic stress syndrome. ${ }^{35-37}$ To lower the risk for those serious consequences, appropriate treatment is desirable. If anxiety does also have a detrimental effect on the birth process, there is all the more reason for screening early in pregnancy for anxiety. Screening for anxiety early in pregnancy provides sufficient time for treatment or therapy for women with high anxiety levels.

Therefore, this study investigates the association between general anxiety and/or pregnancy-related anxiety measured during the first half of pregnancy and the birth process, and the interaction of this association with parity, ethnicity and the level of care at the start of labour.

\section{METHODS}

\section{Study design and participants}

Data were derived from the Amsterdam Born Children and their Development (ABCD) study, a large multiethnic prospective cohort study. ${ }^{38}$ The ABCD study is aimed at examining the relationship between maternal lifestyle and psychosocial conditions during pregnancy and the child's health at birth as well as later in life. Between January 2003 and March 2004, participating obstetric care providers (midwives and hospital obstetricians; participation rate 96\%) invited pregnant women in Amsterdam at their first antenatal visit to enrol in the ABCD. A total of 12373 women were approached (99\% of the target population). Within 2 weeks of this first contact, women who agreed to take part were sent a questionnaire, covering sociodemographic conditions, obstetric history and psychosocial conditions, including general and pregnancy-related anxiety. Consent forms were also sent for the linkage of study data to medical records as well as to data from the Dutch Perinatal Registration (PRN).

A total of 8266 women (response rate $67 \%$ ) filled in the pregnancy questionnaire at an average of 16 weeks gestation (IQR 14-18 weeks), and 7043 gave permission for perusal of their medical records. Three months after birth, the women who gave permission for follow-up $(\mathrm{n}=6854)$ received a questionnaire concerning, among other things, the course of pregnancy and delivery. A total of 5218 mothers filled out this questionnaire. ${ }^{38}$ To facilitate participation by women unable to speak Dutch, questionnaires were also available in Turkish, Arabic and English, and women could also complete the questionnaire with the assistance of an interviewer. Participation of foreign-born women was lower $(42 \%-64 \%)$ compared with Dutch-born women (77\%) but comparable with response rates in other population-based, multiethnic studies in the Netherlands. ${ }^{39}$ In the present study, we included women with a singleton pregnancy, a gestational age $\geq 24$ weeks and a living fetus at the start of labour.

Approval for the study was obtained from the Medical Ethical Committees of the participating hospitals and the Registration Committee of the Municipality of Amsterdam. All women gave written informed consent.

\section{Definition and measurement of variables}

\section{General and pregnancy-related anxiety}

General anxiety was assessed using the Dutch version of the State-Trait Anxiety Inventory (STAI). ${ }^{40} 41$ The 20 items regarding the state anxiety subscale were included in our questionnaire, with each item scored on a 4-point scale. The anxiety score was dichotomised in low-average $(<43$ points) and high anxiety ( $\geq 43$ points). ${ }^{42}$ In our sample, the internal consistency (Cronbach's alpha) of the scale was 0.94 .

Pregnancy-related anxiety was assessed using an abbreviated 10-item version of the Pregnancy-Related Anxieties Questionnaire (PRAQ). ${ }^{43}$ The internal consistency (Cronbach's alpha) was 0.79 ; each item was scored on a 4-point scale. Three aspects that can be distinguished in the PRAQ are 'fear of labour', 'fear of bearing a physically or mentally handicapped child' (hereafter 'fear of child') and 'concern about one's appearance'. In the present study, our focus was on the total score on pregnancy-related anxiety. One question was only applicable for nulliparous women ('I am scared of labour and birth because I have never experienced this'), resulting in a maximum score of 40 for nulliparous women and 36 for multiparous women. Because cut-off scores were not available for dichotomisation of the results from 
this instrument, we used the 90th percentile to identify women with a high level of pregnancy-related anxiety. ${ }^{45}$ This resulted in cut-off scores of 28 and 24 for nulliparous and multiparous women, respectively. We performed additional explorative analyses, using the PRAQ subscales 'fear of labour' (only for nulliparous women) and 'fear of child' These were dichotomised based on the 10th percentile; the cut-offs for 'fear of labour' and 'fear of child' were 10 and 12 , respectively.

\section{Birth process and interventions}

Outcome data on the birth process and interventions were obtained by linking our records with the PRN database. Validation of the PRN database has been described previously. ${ }^{39}$ The outcome variables were determined from the records of the Registry of Midwives, the Registry of Obstetricians and the Registry of Paediatricians according to the decision rules of the PRN. ${ }^{46}$

We defined the following outcomes:

- Birth process: duration of first stage of labour ( $\leq 12$ hours/>12 hours; only available in registry of midwives) and duration of second stage $(<1.5$ hours $/ \geq 1.5$ hours $)]$.

- Interventions: primary caesarean (yes/no), induction of labour (yes/no), referral during labour (only in the group that started labour under primary care (yes/no)), augmentation (yes/no), pain relief/ sedation (yes/no), secondary caesarean (yes/ no) and vaginal instrumental delivery (ventouse or forceps (yes/no)).

\section{Covariates}

The following covariates were included in the analyses: maternal age (years), ethnicity, education (years after primary school), prepregnancy BMI $\left(\mathrm{kg} / \mathrm{m}^{2}\right)$, parity (nulliparae/multiparae), smoking during pregnancy (yes/no), alcohol use during pregnancy (yes/no), hypertensive disorders and diabetes (pre-existent or detected during pregnancy), gestational age at delivery (weeks), care at start of labour (primary/secondary) and birth weight $(\mathrm{g})$. Ethnicity was based on the birth country of the participant's mother (self-reported) and included the following categories: Dutch, Turkish, Moroccan, Black (Antillean/Aruban, Surinamese, Ghanaian or other African descent), other non-Western and other Western countries. Prepregnancy weight and height, education, smoking and alcohol use during pregnancy were self-reported in the questionnaire. Parity, birth weight, gestational age, hypertension and diabetes were extracted from the PRN database. Missing data concerning parity, birth weight and gestational age were extracted from the 3-month questionnaire; hypertension and diabetes were encoded as 'yes' if these diseases were reported in the PRN database and/or in the 3-month questionnaire.

\section{Statistical analysis}

Descriptive statistics were used to profile the sample characteristics according to the level of maternal general anxiety and of pregnancy-related anxiety. Categorical variables were described by percentages per category. Continuous variables were described using the mean and $95 \% \mathrm{CI}$ if normally distributed and, if not, with the median and minimum and maximum values. Risks were presented as OR and 95\% CI. Differences were tested with Student's t-test or Mann-Whitney U test for continuous variables and Pearson's $\chi^{2}$ test for categorical variables. Associations between high general and/or pregnancy-related anxiety compared with low anxiety were analysed for each outcome using multiple logistic regression analyses. All potential confounders were determined a priori and added to the regression model. We adjusted for general covariates: age, ethnicity, education, prepregnancy BMI, parity, smoking and alcohol use. To avoid overadjustment, we did not adjust for potential intermediate variables, such as hypertension, diabetes, gestational age and birth weight. Subgroup analyses were performed according to parity (nulliparous vs multiparous women), ethnicity (Dutch, Turkish, Moroccan and Black) and care at start of labour (primary/secondary care). No subgroup analysis was performed in the ethnic groups 'other Western' and 'other non-Western' as each of these groups represented a diverse selection of ethnicities. To formally test whether different associations existed for the different subgroups (parity, ethnicity and care at start of labour) between STAI or PRAQ and the birth process variables, interaction terms (between parity, ethnicity respectively care at start of labour with general and pregnancy-related anxiety) were added to the final model. We performed additional explorative analyses, using the PRAQ subscales 'fear of labour' (only for nulliparous women) and 'fear of child' following the same procedure.

All analyses were performed with SPSS 21.0.

\section{RESULTS}

\section{Response}

Of the 8266 women who completed the pregnancy questionnaire, 6616 women had valid results for the STAI or PRAQ and outcome data. The mean STAI score was significantly higher in the group of women with missing outcome data (39.5 vs 38.2); the PRAQ scores were comparable. After exclusion of 173 women (with a multiple fetus, a gestational age $\leq 24$ weeks or an antenatal death), 6443 records were available for analysis (figure 1). Most women completed both the STAI and the PRAQ ( $\mathrm{n}=6335)$; 37 women completed only the STAI and 71 women only the PRAQ.

\section{Covariates according to anxiety levels}

A high STAI score (further referred to as 'high general anxiety') was found in $30.9 \%$ of the sample and a high score on the PRAQ (further referred to as 'high pregnancy-related anxiety') in $11.1 \%$ of the sample. The STAI score was moderately correlated with the PRAQ score (Pearson's $\mathrm{r}=0.36 ; \mathrm{p}<0.001$ ). 


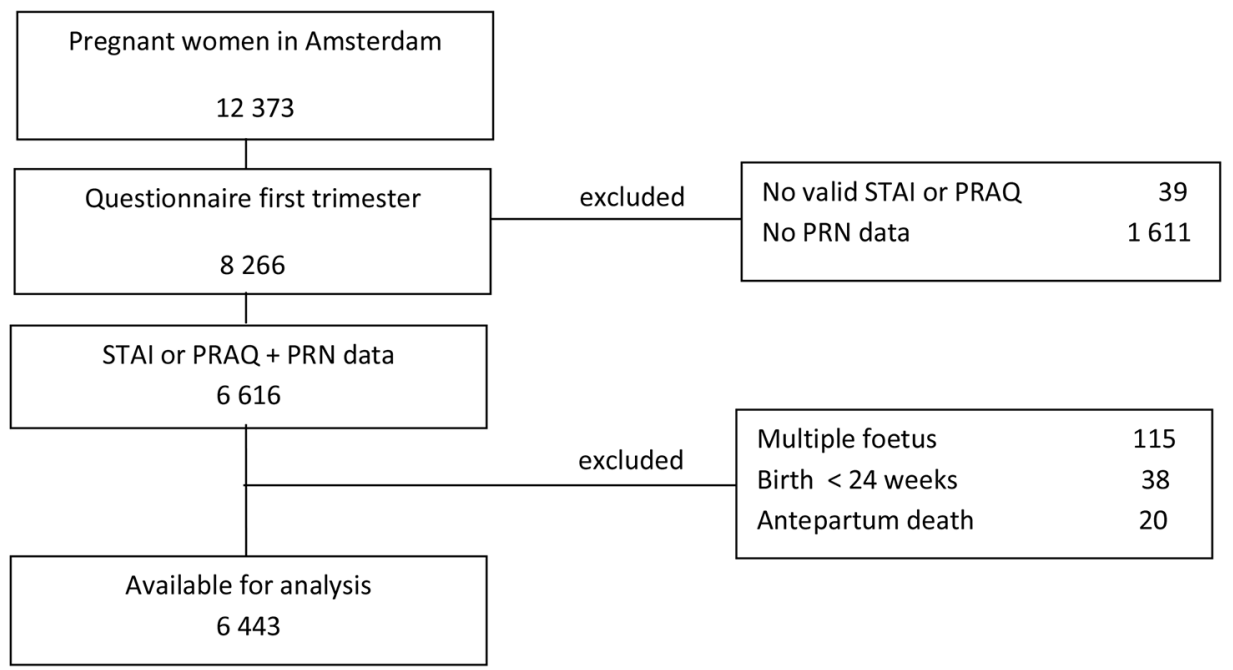

STAI = State Trait Anxiety Inventory

PRAQ = Pregnancy-Related Anxiety Questionnaire

PRN=Dutch Perinatal Registration

Figure 1 Flowchart of the Amsterdam Born Children and their Development study and inclusion in the current analyses.

High general anxiety and high pregnancy-related anxiety were more frequently observed in younger women, in women with non-Dutch ethnicities, fewer years of education, higher prepregnancy BMIs, smoking women and less alcohol consumption and in women who gave birth to babies with lower birth weight. There were no differences in the rates of hypertension and gestational age between the high and the low anxiety groups; however, diabetes and secondary care at the start of labour were more frequently observed in women with high general anxiety. Nulliparous women appeared to be at lower risk for high general anxiety compared with multiparous women (table 1).

\section{Outcome prevalences}

The prevalences of the indicators of the birth process are shown in table 2.

Most prevalences were higher $(\mathrm{p}<0.05)$ in nulliparous than in multiparous women, except primary caesarean section $(p=0.09)$. Most prevalences were similar in the different ethnicities, except for a higher prevalence of prolonged second stage of labour and instrumental delivery in Dutch women $(\mathrm{p}<0.001)$.

\section{Multivariable analyses}

General anxiety and parity

After adjustment, women with high general anxiety were more likely to receive pain relief/sedation, in which association was only significant in nulliparous women. Multiparous women were more likely to undergo induction of labour, which was not seen in nulliparae (table 2). Moreover, statistically significant interactions between parity and general anxiety were found for primary caesarean section and a second stage of $\geq 90 \mathrm{~min}$ : highly anxious multiparous women were more likely to experience these two outcomes, compared with non-anxious women, whereas highly anxious nulliparous women were at decreased risk for these outcomes.

\section{Pregnancy-related anxiety and parity}

After adjustment, women with high pregnancy-related anxiety were more likely to receive pain relief/sedation, which association was only significant in nulliparous women. Subgroup analysis showed an increased risk for primary caesarean in highly anxious multiparous women; however, no statistically significant interactions with parity were observed when formal interaction testing was completed (table 2).

Analyses with the subscales showed similar trends with one exception: fear of labour was associated with an increased risk for pain relief/sedation in (nulliparous) women, which was not the case for high 'fear of child' (see online supplementary table 1).

\section{General and pregnancy-related anxiety and ethnicity}

After adjustment, no statistically significant interactions were found between general or pregnancy-related anxiety with ethnicity for any of the outcome measures (all $\mathrm{p}$ values $\geq 0.12$ ). The increased risk for pain relief/ sedation was only significant in Dutch women with high general or pregnancy-related anxiety.

However, subgroup analyses suggested a statistically significant lower risk for primary caesarean in Moroccan women with high general anxiety and an increased risk for instrumental delivery in Moroccan women with high general anxiety and in Turkish women with high pregnancy-related anxiety (table 3 ). 


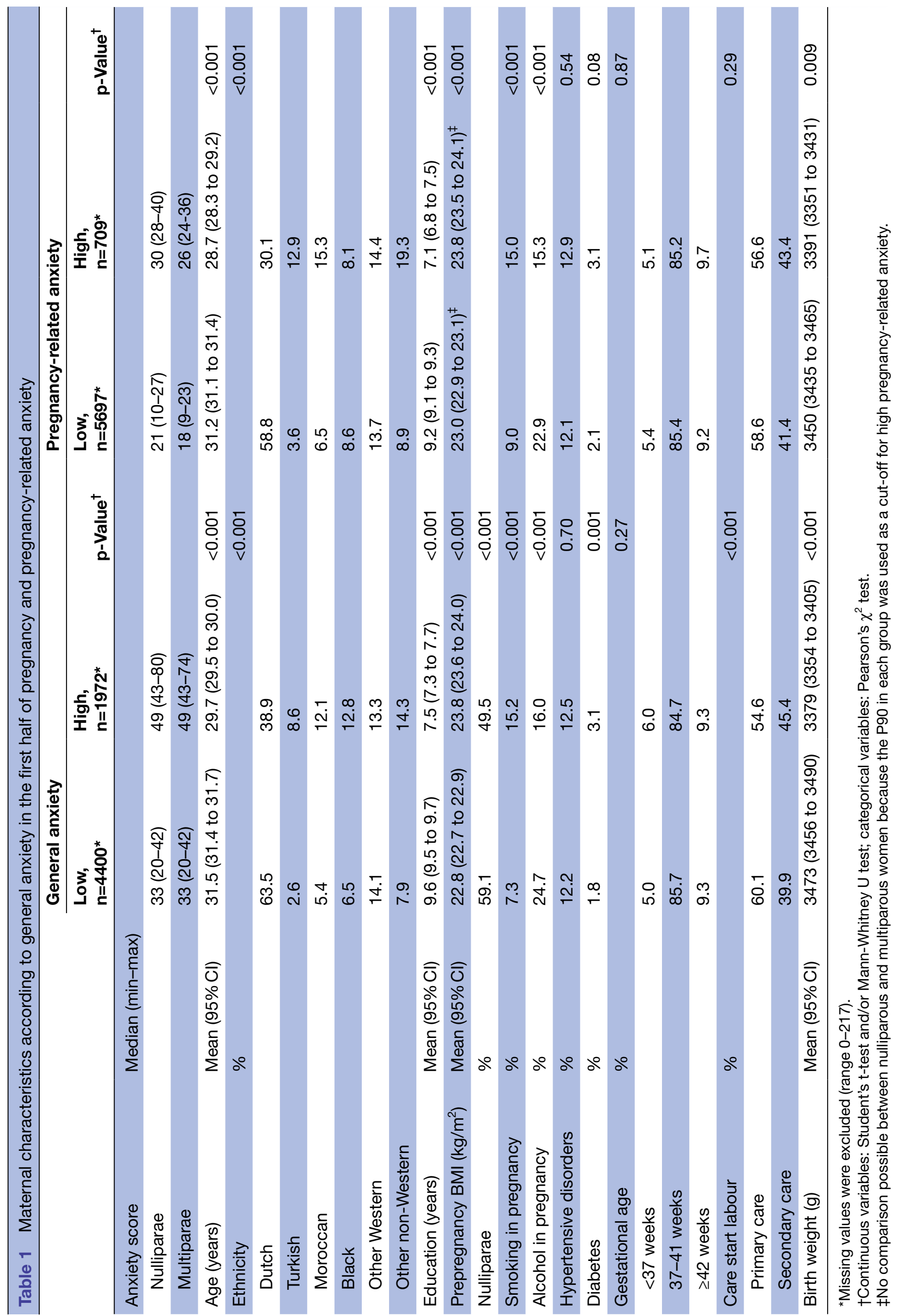

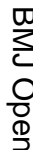

竞

음

จิ

is

우

$\vec{\omega}$

응.

党

옹

$\stackrel{\circ}{\omega}$

$\frac{\sharp}{\omega}$

옥

文

옥

ఫ

응

$\stackrel{2}{\circ}$

옹

룽

호․

음

홍.

옹

음

을

N

N

ए

$\stackrel{0}{0}$

$\stackrel{\mathbb{\Phi}}{\mathscr{Q}}$

뭉

迥

एक

음 
Table 2 Univarible and multivariable associations between general and pregnancy-related anxiety in the first half of pregnancy and (interventions in) the birth process, according to parity

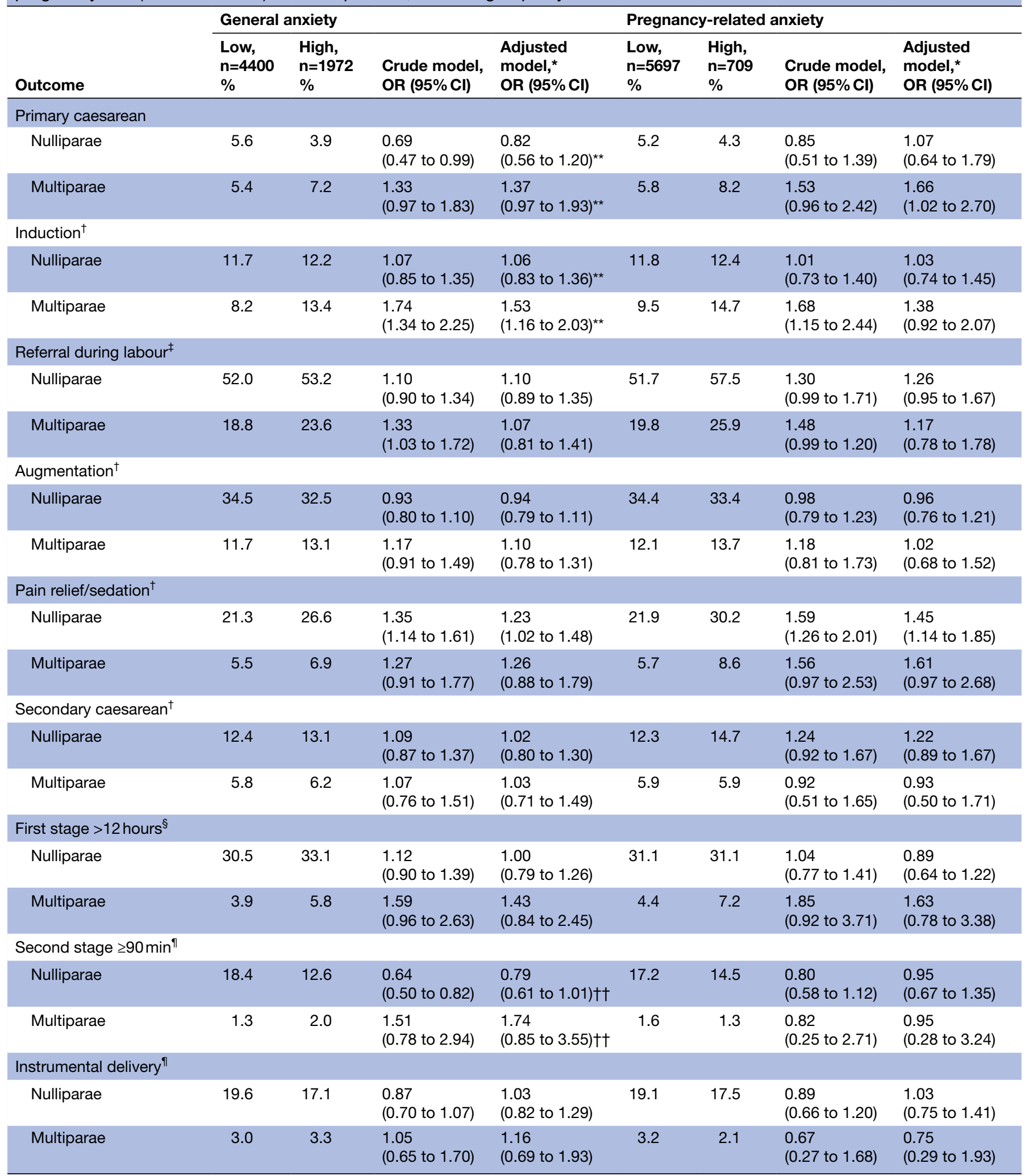

*Adjusted for body mass index, maternal age, years of education, ethnicity, smoking and alcohol.

†Excluded women with primary caesarean section.

$\ddagger$ Only women under primary care at start of labour.

§Excluded women with caesarean section; data available for 3533 women (only recorded in perinatal registry of midwives).

IExcluded women with caesarean section (primary/ secondary).

${ }^{* *} p$ value interaction parity and anxiety 0.018 .

$\dagger † p$ value interaction parity and anxiety 0.017 . 


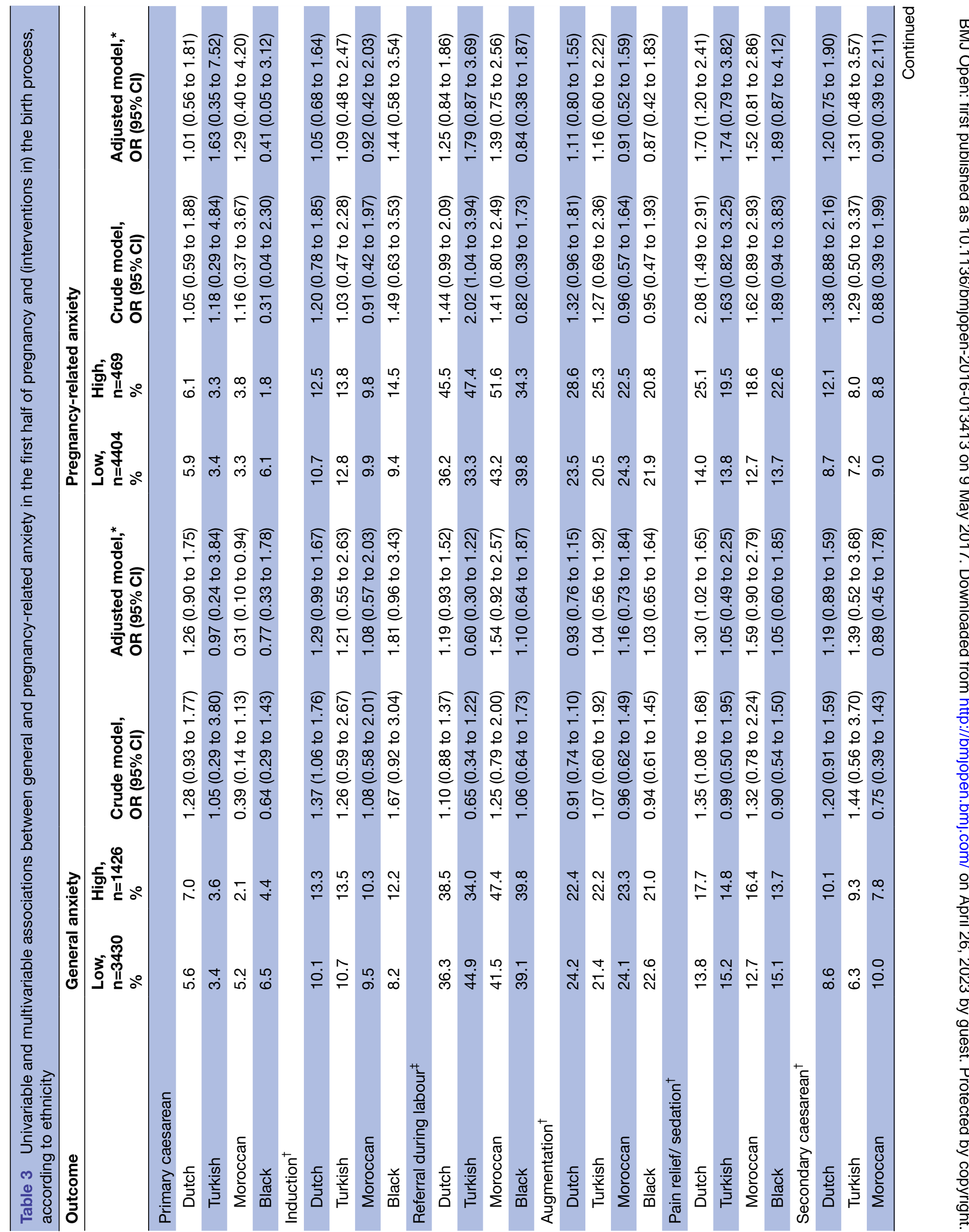




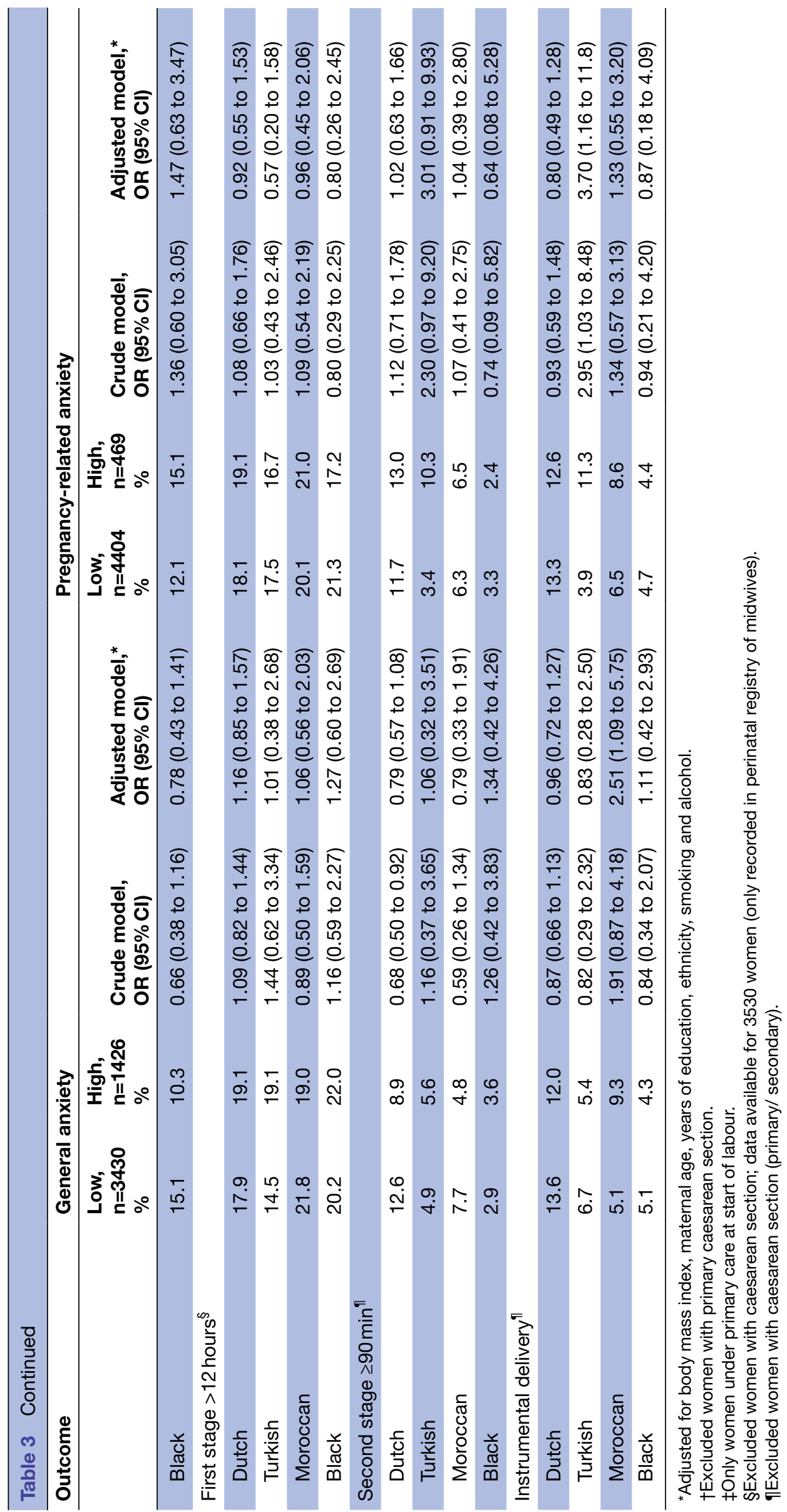


Analyses with the subscales showed similar trends. However, in Dutch women with high 'fear of labour', the risk for a first stage of labour longer than 12 hours and for a second stage of labour longer than 1.5 hours was increased. High 'fear of child' was not associated with an increased risk for any of the outcome parameters (see online supplementary table 2 ).

\section{General and pregnancy-related anxiety and level of care}

No statistically significant interactions were found between general or pregnancy-related anxiety with the level at care at the start of labour for any of the outcome measures (all $p$ values $\geq 0.07$ ). However, in the subgroup analyses, the risk for pain relief/sedation was increased in highly anxious women who started labour in secondary care only (general anxiety: OR 1.26; 95\% CI 1.004 to 1.58 ; pregnancy-related anxiety: OR $1.81 ; 95 \%$ CI 1.33 to 2.45 ) compared with those who began labour in primary care (general anxiety: OR 1.09; 95\% CI 0.84 to 1.40 ; pregnancy-related anxiety: OR 1.17 ; $95 \%$ CI 0.84 to 1.63 ) (see online supplementary table 3 ).

The subscale analyses showed that fear of labour was associated with pain relief/sedation, which was not the case of high 'fear of child' (see online supplementary table 4).

\section{DISCUSSION}

\section{Main findings}

In this multiethnic cohort, general anxiety and pregnancy-related anxiety, as measured in the first half of pregnancy, were not associated with the progress of the birth process but were associated with an increased risk for interventions during labour. Pregnancy-related anxiety showed stronger associations than general anxiety. Some associations differed between nulliparous and multiparous women, whereas largely similar associations were found across all ethnic groups.

\section{Strengths and limitations}

A major strength of the present study is the prospective design in a large multiethnic cohort. Although the participation of non-Dutch women was lower than that of Dutch women, we collected data for $\geq 2800$ non-Dutch women, making it possible to perform subgroup analyses in the four major ethnic groups in the Netherlands. However, the sample sizes were not large enough to prove a potential interaction effect of ethnicity and anxiety with less frequent outcomes. Although our sample might not be representative for all pregnant women in Amsterdam or in the Netherlands, there is no reason to assume that the association between anxiety and the birth process should be different in non-responders or in women with missing outcome data.

Another strength is the assessment of both general anxiety and pregnancy-related anxiety, using validated questionnaires, whereas other studies only assessed pregnancy-related anxiety.
A limitation of the abbreviated 10 -item version of the PRAQ that we used is that this questionnaire has only been validated in nulliparous women. Because one item of the three-item subscale 'fear of labour' was not applicable for multiparae, the assessment of this aspect in multiparae was limited. Therefore, we used the total PRAQ score but also performed additional analyses to explore the aspects 'fear of labour' (only in nulliparous women) and 'fear of getting a handicapped child'. The use of the total PRAQ score is in agreement with other questionnaires, such as the Wijma Delivery Expectancy Questionnaire (a frequently used questionnaire) conceptualised as a unidimensional instrument to measure fear of childbirth. ${ }^{47}$ Outcome data on the birth process and interventions were available from the PRN, as well as from a questionnaire filled out 3 months after birth by the mother. Combination of these two data sources resulted in a limited amount of missing data within the group with outcome data available, and there is no reason to assume that data were missing in a selective manner. Since the collection of our data in 2003-2004, the prevalence of interventions during labour has increased. ${ }^{1-3} 48$ Nevertheless, we think that the associations between anxiety and the birth process have remained similar.

Anxiety was measured in the first half of pregnancy, whereas other studies measured anxiety later in pregnancy. ${ }^{16}$ Little evidence is available concerning how anxiety in the first half of pregnancy persists and evolves during pregnancy, but in case of fear of childbirth/ labour, it might be possible that fear will increase during pregnancy because the event that is feared is unavoidable and slowly coming closer. Moreover, assessment of anxiety in the first half of pregnancy provides the opportunity to perform an intervention to reduce anxiety, particularly pregnancy-related anxiety.

\section{Comparison with other studies}

The prevalence of high general anxiety was higher in our study than in other Dutch studies. ${ }^{49} 50$ This can be explained by the large proportion of women of non-Dutch origin in our study.

Some studies found no association between general anxiety and (interventions in) the birth process, ${ }^{16}{ }_{51}$ whereas Adams $e t$ al were the only group to find a trend for increased risk for labour induction in general anxious women in a cohort of 2206 women. ${ }^{14}$ To our knowledge, our study is the first to show a statistically significant association between general anxiety and interventions during birth. The difference between our study and others might be explained by a lack of power in other studies (including 88 and 1515 women, respectively), as well as by different views on the management of labour, for example, the use of pain relief. Moreover, the other studies did not investigate the association between anxiety and labour induction, as this is not considered to be an 'undesirable' outcome.

Our observed association between pregnancy-related anxiety and pain relief/sedation is in line with other studies reporting an association between fear of labour 
and epidural analgesia. ${ }^{1752}$ Several studies found an association between fear of labour and elective caesarean section. ${ }^{18-21}$ Unfortunately, in our data, elective caesarean was not coded, but only primary caesarean. Although we found no association between general or pregnancy-related anxiety and primary caesarean, except in the subgroup of Moroccan women, an interaction was seen between parity and general anxiety, suggesting a decreased risk for primary caesarean in anxious nulliparae and an increased risk in anxious multiparae. This might reflect the restraint of obstetricians in the Netherlands to perform an elective caesarean in nulliparae. In anxious multiparae with a complicated birth history, obstetric caregivers will be more willing to perform a caesarean section. Although others reported an association between fear of labour and emergency caesarean section, this was not seen in our study. ${ }^{15} 23$

Several studies found a longer duration of labour in women with high fear of labour. ${ }^{14-16}$ We did not find such an association with general or pregnancy-related anxiety, but we did find an association with the PRAQ subscale 'fear of labour' within the subgroup of nulliparous Dutch women. However, this result from an explorative analysis should be interpreted with caution. Unfortunately, in our study, data on the duration of the first stage of labour were only available in categories of 6 hours. Since we did not find an association with augmentation during labour or artificial delivery, we think that an association with the process of birth in our population is very unlikely.

Subgroup analyses showed some different effects in nulliparae and multiparae. Parity is known to be associated with the birth process, which was confirmed in the present study. ${ }^{81011}$ The different effect of anxiety on interventions in the birth process in nulliparae and multiparae can be explained by physiological factors, by the woman's experience during the previous labour, resulting in more anxiety in women with a complicated previous labour, and also by the information available for the care provider on the previous birth process. The increased risk for induction of labour in multiparae with high general anxiety may be due to greater confidence by obstetric caregivers that the induction will be successful in multiparae. ${ }^{5354}$ An increased risk for referral during labour was only seen in nulliparous women with high 'fear of labour', an aspect of pregnancy-related anxiety. This can be explained by the lower a priori risk for referral in multiparae and the subsequent greater confidence of primary care midwives in a successful delivery in primary care. To our knowledge, this is the first study to investigate possible interactions with parity with regard to the impact of anxiety on the birth process. More research is necessary to unravel the factors contributing to the different effects of anxiety in nulliparous and multiparous women.

In contrast to our expectation, we found no stronger associations between general or pregnancy-related anxiety in the non-Dutch groups compared with the Dutch group. However, some indication was found for an increased risk for instrumental delivery in Turkish women with high pregnancy-related anxiety, for Moroccan women with high general anxiety and for a lower risk for primary caesarean in highly anxious Moroccan women. It is unlikely that these findings are explained by the lack of prenatal healthcare visits or language barriers because these factors are also seen among Moroccan and Surinamese women. ${ }^{55}$ However, these latter findings should be interpreted with caution as the subgroups were small; we recommend more studies in large multiethnic cohorts to address these questions. The large prevalence of high anxiety in non-Dutch women also justifies this research.

Some indications were found that the odds for pain relief/sedation were especially increased in anxious women who started labour in secondary care compared with primary care. This may reflect that primary caregivers use different strategies to support anxious women during labour.

It is reassuring that we found no association between general and pregnancy-related anxiety and the progression of birth, but only with interventions. Another Dutch study showed that referral during labour, a significant intervention within the Dutch context, was associated with a negative recall of labour 3 years late. ${ }^{56}$ In 2013, the need for pain relief was the most frequent reason $(18.5 \%)$ for referral during labour. ${ }^{57}$ Therefore, it is worthwhile to investigate whether these interventions during labour can be managed by treatment or therapy during pregnancy in anxious women, to avoid the necessity of labour-related interventions. If effective treatments are identified, screening for anxiety (especially pregnancy-related anxiety showing the strongest associations) should be implemented in prenatal care, at least in subgroups with an increased risk for anxiety. The observed high prevalence of general and pregnancy-related anxiety in non-Dutch women underlines the relevance of screening and subsequent fear-reducing interventions, especially in this group.

\section{CONCLUSION}

General and pregnancy-related anxiety in the first half of pregnancy are not associated with prolonged or obstructed labour and artificial delivery and, therefore, do not appear to influence the progression of birth. However, high levels of anxiety contribute to greater use of interventions during labour, especially pain relief/sedation, induction of labour and possibly referral during labour. Although we found similar associations between ethnic groups, the high prevalence of anxiety symptoms in pregnant women with a migrant background justifies more research on the effect of interventions to reduce anxiety symptoms in ethnic groups.

Acknowledgements The authors would like to thank all participants of the ABCD study. They also thank Catherine Barrett for developing the first design of the study.

Contributors The study was designed by JMK and TGMV. JMK performed the statistical analysis and drafted the manuscript. TGMV rendered and provided the ABCD Study data. JMK, TGMV and AMS interpreted the results of the analysis. TGMV and AMS made substantial contributions in revising the manuscript. The final manuscript was read and approved by all authors. 
Competing interests None declared.

Ethics approval Approval for the ABCD study was obtained from the Central Committee on Research involving Human Subjects in the Netherlands, the Medical Ethical Committees of participating hospitals and the Registration Committee of Amsterdam.

Provenance and peer review Not commissioned; externally peer reviewed.

Data sharing statement Data of the ABCD study can be shared for specific research questions that are available from the corresponding author on request.

Open Access This is an Open Access article distributed in accordance with the Creative Commons Attribution Non Commercial (CC BY-NC 4.0) license, which permits others to distribute, remix, adapt, build upon this work non-commercially, and license their derivative works on different terms, provided the original work is properly cited and the use is non-commercial. See: http://creativecommons.org/ licenses/by-nc/4.0/

(C) Article author(s) (or their employer(s) unless otherwise stated in the text of the article) 2017. All rights reserved. No commercial use is permitted unless otherwise expressly granted.

\section{REFERENCES}

1. Kwee A, Elferink-Stinkens PM, Reuwer PJ, et al. Trends in obstetric interventions in the Dutch obstetrical care system in the period 19932002. Eur J Obstet Gynecol Reprod Biol 2007;132:70-5.

2. Zhao $\mathrm{Y}$, Zhang J, Hukkelhoven $\mathrm{C}$, et al. Modest rise in caesarean section from 2000-2010: the Dutch experience. PLoS One 2016;11:e0155565.

3. Dutch Perinatal Registration. Perinatal care in the netherlands 2013. Utrecht: Stichting Perinatale Registratie Nederland: Figure 4.1. http:// www.perinatreg.nl/uploads/150/153/PRN_jaarboek_2013_09122014. pdf.

4. Brown HK, Hill J, Natale R. Caesarean section rates in Southwestern Ontario: changes over time after adjusting for important medical and social characteristics. J Obstet Gynaecol Can 2014;36:578-89.

5. Einarsdóttir K, Haggar F, Pereira G, et al. Role of public and private funding in the rising caesarean section rate: a cohort study. BMJ Open 2013;3:e002789.

6. Dahlen HG, Tracy S, Tracy M, et al. Rates of obstetric intervention among low-risk women giving birth in private and public hospitals in NSW: a population-based descriptive study. BMJ Open 2012;2:e001723.

7. Delbaere I, Cammu H, Martens E, et al. Limiting the caesarean section rate in low risk pregnancies is key to lowering the trend of increased abdominal deliveries: an observational study. BMC Pregnancy Childbirth 2012;12:3.

8. Schuit E, Kwee A, Westerhuis ME, et al. A clinical prediction model to assess the risk of operative delivery. BJOG 2012;119:915-23.

9. Daemers DO, Wijnen HA, van Limbeek EB, et al. The impact of obesity on outcomes of midwife-led pregnancy and childbirth in a primary care population: a prospective cohort study. BJOG 2014;121:1403-14.

10. Essex HN, Green J, Baston $\mathrm{H}$, et al. Which women are at an increased risk of a caesarean section or an instrumental vaginal birth in the UK: an exploration within the millennium cohort study. BJOG 2013;120:732-43.

11. Sandall J, Soltani H, Gates S, et al. Midwife-led continuity models versus other models of care for childbearing women. Cochrane Database Syst Rev 2013;8:CD004667.

12. Alder J, Fink N, Bitzer J, et al. Depression and anxiety during pregnancy: a risk factor for obstetric, fetal and neonatal outcome? A critical review of the literature. J Matern Fetal Neonatal Med 2007;20:189-209.

13. Johnson RC, Slade P. Obstetric complications and anxiety during pregnancy: is there a relationship? J Psychosom Obstet Gynaecol 2003;24:1-14

14. Adams SS, Eberhard-Gran M, Eskild A. Fear of childbirth and duration of labour: a study of 2206 women with intended vaginal delivery. BJOG 2012;119:1238-46.

15. Laursen $M$, Johansen $C$, Hedegaard M. Fear of childbirth and risk for birth complications in nulliparous women in the danish national birth cohort. BJOG 2009;116:1350-5.

16. Reck C, Zimmer K, Dubber S, et al. The influence of general anxiety and childbirth-specific anxiety on birth outcome. Arch Womens Ment Health 2013;16:363-9.

17. Hall WA, Stoll K, Hutton EK, et al. A prospective study of effects of psychological factors and sleep on obstetric interventions, mode of birth, and neonatal outcomes among low-risk British Columbian women. BMC Pregnancy Childbirth 2012;12:78.

18. Melender HL. Fears and coping strategies associated with pregnancy and childbirth in Finland. J Midwifery Womens Health 2002;47:256-63.

19. Graham WJ, Hundley V, McCheyne AL, et al. An investigation of women's involvement in the decision to deliver by caesarean section. Br J Obstet Gynaecol 1999;106:213-20.

20. Nieminen K, Stephansson O, Ryding EL. Women's fear of childbirth and preference for cesarean section-a cross-sectional study at various stages of pregnancy in Sweden. Acta Obstet Gynecol Scand 2009;88:807-13.

21. Handelzaltss J, Fisher S, Lurie S, et al. Fear of childbirth and caesarean delivery on demand. Acta Obstet Gynecol Scand 2012;92:16-21.

22. Ryding EL, Lukasse M, Parys AS, et al; Bidens Group. Fear of childbirth and risk of cesarean delivery: a cohort study in six European countries. Birth 2015;42:48-55.

23. Ryding EL, Wijma B, Wijma K, et al. Fear of childbirth during pregnancy may increase the risk of emergency cesarean section. Acta Obstet Gynecol Scand 1998;77:542-7.

24. Sluijs AM, Cleiren MP, Scherjon SA, et al. No relationship between fear of childbirth and pregnancy-/delivery-outcome in a lowrisk Dutch pregnancy cohort delivering at home or in hospital. $J$ Psychosom Obstet Gynaecol 2012;33:99-105.

25. Johnson $R$, Slade $P$. Does fear of childbirth during pregnancy predict emergency caesarean section? BJOG 2002;109:1213-21.

26. Fenwick J, Gamble J, Nathan E, et al. Pre- and postpartum levels of childbirth fear and the relationship to birth outcomes in a cohort of Australian women. J Clin Nurs 2009;18:667-77.

27. Giscombé CL, Lobel M. Explaining disproportionately high rates of adverse birth outcomes among African Americans: the impact of stress, racism, and related factors in pregnancy. Psychol Bull 2005;131:662-83.

28. Barnes VA, Treiber FA, Musante L, et al. Ethnicity and socioeconomic status: impact on cardiovascular activity at rest and during stress in youth with a family history of hypertension. Ethn Dis 2000;10:4-16.

29. Dysart JM, Treiber FA, Pflieger K, et al. Ethnic differences in the myocardial and vascular reactivity to stress in normotensive girls. Am $J$ Hypertens 1994;7:15-22.

30. Hogue CJ, Bremner JD. Stress model for research into preterm delivery among black women. Am J Obstet Gynecol 2005;192:S47-S55.

31. Schrier AC, de Wit MA, Coupé VM, et al. Comorbidity of anxiety and depressive disorders: a comparative population study in western and non-Western inhabitants in the Netherlands. Int $J$ Soc Psychiatry 2012;58:186-94.

32. Garrett PW, Dickson HG, Young L, et al. "The Happy Migrant Effect": perceptions of negative experiences of healthcare by patients with little or no English: a qualitative study across seven language groups. Qual Saf Health Care 2008;17:101-3.

33. Lindert J, Ehrenstein OS, Priebe S, et al. Depression and anxiety in labor migrants and refugees - a systematic review and metaanalysis. Soc Sci Med 2009;69:246-57.

34. Levecque K, Lodewyckx I, Vranken J. Depression and generalised anxiety in the general population in Belgium: a comparison between native and immigrant groups. J Affect Disord 2007;97:229-39.

35. Dunkel Schetter C, Tanner L. Anxiety, depression and stress in pregnancy: implications for mothers, children, research, and practice. Curr Opin Psychiatry 2012;25:141-8.

36. Glynn LM, Schetter CD, Hobel CJ. Sandman CA. Health Psychology 2008;27:43-51.

37. Van den Bergh BR, Mulder EJ, Mennes M, et al. Antenatal maternal anxiety and stress and the neurobehavioural development of the fetus and child: links and possible mechanisms. a review. Neurosci Biobehav Rev 2005;29:237-58.

38. van Eijsden M, Vrijkotte TG, Gemke RJ, et al. Cohort profile: the Amsterdam Born Children and their Development (ABCD) study. Int $J$ Epidemiol 2011;40:1176-86.

39. Tromp M, van Eijsden M, Ravelli AC, et al. Anonymous non-response analysis in the ABCD cohort study enabled by probabilistic record linkage. Paediatr Perinat Epidemiol 2009;23:264-72.

40. der PHMvan, Defares PB, Spielberger CD. [Een nederlandstalige bewerking van de spielberger State-Trait Anxiety Inventory: de ZelfBeoordelings vragenlijst]. De Psycholoog 1980;15:460-7.

41. Spielberger CD, Gorsuch RL, Lushene RE. STAl manual for the State-Trait Anxiety Inventory. Palo Alto, CA: Consulting Psychologists Press, 1970.

42. Mennes M, Stiers $P$, Lagae L, et al. Long-term cognitive sequelae of antenatal maternal anxiety: involvement of the orbitofrontal cortex. Neurosci Biobehav Rev 2006;30:1078-86. 
43. Huizink AC, Mulder EJ, Robles de Medina PG, et al. Is pregnancy anxiety a distinctive syndrome? Early Hum Dev 2004;79:81-91.

44. Van den Bergh $B$. The influence of maternal emotions during pregnancy on fetal and neonatal behavior. Pre- and Perinatal Psychology J 1990;5:119-13.

45. Loomans EM, van Dijk AE, Vrijkotte TG, et al. Psychosocial stress during pregnancy is related to adverse birth outcomes: results from a large multi-ethnic community-based birth cohort. Eur J Public Health 2013;23:485-91.

46. Goossen-Baremans A. Consensusdocument beslisregels dataset PRN. versie 0.99. Utrecht: Stichting Perinatale Registratie Nederland 2009. http://www.perinatreg.nl/publicaties?noCache= 602;1459936592.

47. Wijma K, Wijma B, Zar M. Psychometric aspects of the W-DEQ; a new questionnaire for the measurement of fear of childbirth. $J$ Psychosom Obstet Gynaecol 1998;19:84-97.

48. Amelink-Verburg MP, Rijnders ME, Buitendijk SE. A trend analysis in referrals during pregnancy and labour in Dutch midwifery care 1988-2004. BJOG 2009;116:923-32.

49. Koelewijn JM, Vrijkotte TG, de Haas M, et al. Women's attitude towards prenatal screening for red blood cell antibodies, other than RhD. BMC Pregnancy Childbirth 2008;8:49.
50. Fontein-Kuipers $Y$, Ausems M, Budé L, et al. Factors influencing maternal distress among Dutch women with a healthy pregnancy. Women Birth 2015;28:e36-e43.

51. Perkin MR, Bland JM, Peacock JL, et al. The effect of anxiety and depression during pregnancy on obstetric complications. Br J Obstet Gynaecol 1993;100:629-34.

52. Thomson M, Hanley J. Factors predisposing to difficult labor in primiparas. Am J Obstet Gynecol 1988;158:1074-8.

53. Bricker L, Luckas M. Amniotomy alone for induction of labour. Cochrane Database Syst Rev 2000. CD002862.

54. Howarth GR, Botha DJ. Amniotomy plus intravenous oxytocin for induction of labour. Cochrane Database Syst Rev 2001. CD003250.

55. Alderliesten ME, Vrijkotte TG, van der Wal MF, et al. Late start of antenatal care among ethnic minorities in a large cohort of pregnant women. BJOG 2007;114:1232-9.

56. Rijnders $\mathrm{M}$, Baston $\mathrm{H}$, Schönbeck $\mathrm{Y}$, et al. Perinatal factors related to negative or positive recall of birth experience in women 3 years postpartum in the netherlands. Birth 2008;35:107-16.

57. Dutch Perinatal Registration. Perinatal care in the netherlands 2013. [Internet] Utrecht: Stichting Perinatale Registratie Nederland 2014, Table 9.3.1. http://www.perinatreg.nl/uploads/150/153/PRN jaarboek_2013_09122014.pdf. 